\title{
Selectin-mucin interactions as a probable molecular explanation for the association of Trousseau syndrome with mucinous adenocarcinomas
}

\author{
Mark Wahrenbrock, ${ }^{1,3}$ Lubor Borsig, ${ }^{1,2}$ Dzung Le, ${ }^{3}$ Nissi Varki, ${ }^{1,3}$ and Ajit Varki ${ }^{1,2}$ \\ ${ }^{1}$ Department of Medicine, \\ ${ }^{2}$ Department of Cellular \& Molecular Medicine, and \\ ${ }^{3}$ Department of Pathology, Glycobiology Research and Training Center, University of California, San Diego, \\ La Jolla, California, USA
}

\begin{abstract}
Trousseau described spontaneous, recurrent superficial migratory thrombophlebitis associated with occult cancers, and this was later correlated with disseminated microangiopathy (platelet-rich clots in small blood vessels). Trousseau syndrome often occurs with mucinous adenocarcinomas, which secrete abnormally glycosylated mucins and mucin fragments into the bloodstream. Since carcinoma mucins can have binding sites for selectins, we hypothesized that selectin-mucin interactions might trigger this syndrome. When highly purified, tissue-factor free carcinoma mucin preparations were intravenously injected into mice, platelet-rich microthrombi were rapidly generated. This pathology was markedly diminished in P- or L-selectin-deficient mice. Heparin (an antithrombin-potentiating agent that can also block $\mathrm{P}$ - and $\mathrm{L}$-selectin recognition of ligands) ameliorated this platelet aggregation, but had no additional effect in P- or L-selectin-deficient mice. Inhibition of endogenous thrombin by recombinant hirudin also did not block platelet aggregation. Mucins generated platelet aggregation in vitro in hirudinized whole blood, but not in platelet-rich leukocyte-free plasma nor in whole blood from L-selectin-deficient mice. Thus, Trousseau syndrome is likely triggered by interactions of circulating carcinoma mucins with leukocyte L-selectin and platelet P-selectin without requiring accompanying thrombin generation. These data may also explain why heparin ameliorates Trousseau syndrome, while vitamin $\mathrm{K}$ antagonists that merely depress thrombin production do not.
\end{abstract}

J. Clin. Invest. 112:853-862 (2003). doi:10.1172/JCI200318882.

\section{Introduction}

In 1865, Armand Trousseau described recurrent superficial migratory thrombophlebitis preceding the diagnosis of cancer (1), a syndrome to which he himself happened to eventually succumb (2). A classic review of 182 cases of this "paraneoplastic" phenomenon emphasized the formation of venous and arterial platelet-rich microthrombi with secondary microangiopathic hemolytic anemia and a frequent association with mucin-rich adenocarcinomas (3). The term Trousseau syndrome is sometimes used more broadly to refer to any form of excessive coagulation associated with cancer $(2,4-10)$. The underlying pathophysiology is thought to be chronic subclinical disseminated intravascular coag-

Received for publication May 9, 2003, and accepted in revised form July 22, 2003,

Address correspondence to: Ajit Varki, Glycobiology Research and Training Center, University of California, San Diego, 9500 Gilman Drive, Cellular and Molecular Medicine-East Room 1086, La Jolla, California 92093-0687, USA.

Phone: (858) 534-2214; Fax: (858) 534-5611;

E-mail: avarki@ucsd.edu.

Conflict of interest: The authors have declared that no conflict of interest exists.

Nonstandard abbreviations used: tissue factor (TF);

P-selectin-deficient (P-sel ${ }^{-/-}$); L-selectin-deficient (L-sel-/-); periodic acid Schiff (PAS); University of California, San Diego (UCSD); platelet-rich plasma (PRP); mononuclear cells (MNCs); P-selectin glycoprotein ligand-1 (PSGL-1). ulation caused by activated procoagulants. Some studies implicated a factor $\mathrm{X}$-activating cysteine protease $(11-13)$ or tissue factor $(\mathrm{TF})(14,15)$ produced by tumor cells, and a few clinical cases were directly associated with TF production $(4,16)$. Continuous treatment with heparin (a well-known anticoagulant in clinical use) is required to prevent recurrent episodes of thrombosis. Oral anticoagulants (vitamin K antagonists) that also decrease thrombin production are usually ineffective, however $(3,17-21)$. Thus, activation of thrombin-mediated fluid-phase coagulation via $\mathrm{TF}$ and/or the cysteine protease may not be the primary process mediating the classic form of Trousseau syndrome.

The frequent association of Trousseau syndrome with mucin-producing adenocarcinomas also remains unexplained. Mucins are large glycoproteins with clustered O-linked glycans (22-26). Carcinoma cells frequently upregulate expression of a variety of mucin polypeptides such as MUC1, MUC2, MUC5AC, MUC4, and MUC16 $(22,23,25,27-32)$. These are often carriers of sialylated, fucosylated, sulfated glycans and can act as pathological ligands for the selectin family of adhesion molecules (33). Such selectin-mucin interactions are implicated in the hematogenous phase of tumor metastasis (34-36).

Mixtures of abnormal carcinoma mucins (and/or their proteolytic fragments) can be shed by carcinoma cells in significant amounts and can circulate in the bloodstream of cancer patients (32,37-41), often being 
used as prognostic markers $(42,43)$. Thus, it is reasonable to hypothesize that circulating mucins are directly involved in the pathogenesis of Trousseau syndrome. Indeed, some early studies suggested a procoagulant role for mucins $(44,45)$. Contamination of mucin preparations by bioactive lipids and/or by TF (D. Le and S. Rapaport, unpublished observations), however, has confounded any definitive conclusions.

L-, P-, and E-selectins comprise a family of carbohydrate-binding adhesion molecules expressed by leukocytes, platelets, and vascular endothelium (reviewed in refs. 46-49). L-selectin is constitutively expressed on neutrophils, monocytes, and naive lymphocytes. P-selectin is stored in secretory granules of resting platelets and endothelium and rapidly translocated to the cell surface upon activation. E-selectin is newly synthesized in endothelial cells via transcriptional activation initiated by various proinflammatory agonists. While all three selectins recognize structurally related ligands containing sialic acid and fucose residues, optimal ligand formation for L- and P-selectin also requires specifically located sulfate esters (46-48, 50-53).

We and others have shown that heparin can inhibit $\mathrm{P}$ - and L-selectin recognition of ligands (54-59) and that heparin blockade of tumor metastasis is at least partly explained by selectin inhibition, rather than by its anticoagulant activity $(35,36,60)$. Our assumption is that these cell surface mucins mediate direct interactions between the tumor cells and the selectin-bearing blood cells. Considering all the above information, we hypothesized that P-selectin interactions with circulating carcinoma mucins might be involved in Trousseau syndrome. Thus, traces of carcinoma-derived TF might weakly activate the coagulation cascade, generating thrombin, which would then activate platelets, causing them to express P-selectin. The carcinoma mucins could then act as templates to aggregate activated platelets via P-selectin. In testing this hypothesis using TF-free carcinoma mucins intravenously injected into mice, we found that platelet-rich microthrombus formation was dependent not only on P-selectin but also on leukocyte-derived L-selectin. Furthermore, it could occur independently of thrombin generation. Similar findings were obtained using in vitro studies of whole blood. We have shown, we believe for the first time, that L-selectin and P-selectin are involved in a linear reactive pathway triggered by mucins. Our data can explain the classic microangiopathic presentation of Trousseau syndrome associated with mucinproducing carcinomas and the superiority of heparin therapy over vitamin $\mathrm{K}$ antagonists in such cases.

\section{Methods}

Cells and mice. Human colonic adenocarcinoma LS180 cells (ATCC CL 187; American Type Culture Collection, Manassas, Virginia, USA) were cultured in $\alpha$ MEM containing $10 \%$ FBS. Immunodeficient $\mathrm{RAG}^{-/-}$mice (129S6/SvEvTac-Rag2tm1; Taconic Farms, Germantown, New York, USA) and wild-type (C57bl/6J), P-selectin-deficient (P-sel-/-) (B6;129S-Selp $\left.{ }^{\text {tmpHyn}}\right)$, and
L-selectin-deficient (L-sel-/-) (B6;129S-SelltmlHyn) mouse strains were obtained from The Jackson Laboratory (Bar Harbor, Maine, USA) and crossbred to obtain selectin deficiencies in uniform genetic backgrounds (C57bl/6J for at least seven generations), including P-sel ${ }^{-/} / \mathrm{L}_{-} \mathrm{sel}^{-/-}$doubly deficient mice (35).

Purification of carcinoma mucin fragments. Xenograft tumors (0.2-0.8 g wet weight, $1-1.5 \mathrm{~cm}$ diameter) were produced by subcutaneous injection of $2 \times 10^{6} \mathrm{LS} 180$ cells/site into RAG2-/- mice. Pooled tumors (15-30 g wet weight) obtained at approximately 2.5 weeks were homogenized in $50 \mathrm{ml}$ water and centrifuged $(10,000 \mathrm{~g}$, $4^{\circ} \mathrm{C}, 10$ minutes) to remove insoluble debris. Soluble mucins in the supernatant were precipitated by adjustment to pH 5.0 using $100 \mathrm{mM} \mathrm{HCl}$ added in drops, while stirring for 30 minutes on ice. The protein pellet was resolubilized in $25 \mathrm{ml}$ of sterile water containing $0.01 \%$ azide, the solution adjusted to $\mathrm{pH} 7.0$ with $100 \mathrm{mM}$ $\mathrm{NaOH}$ added in drops and subjected to two organic extractions using methanol/chloroform (1:1 vol/vol) at a final ratio of $1: 1 \mathrm{vol} / \mathrm{vol}$. The aqueous phase was adjusted to $25 \mathrm{ml}$ with $50 \mathrm{mM}$ Tris, $100 \mathrm{mM} \mathrm{NaCl}, 100 \mathrm{mM}$ EDTA, $0.01 \% \mathrm{NaN}_{3}$, and repeatedly treated with Tritirachium album proteinase K (Sigma-Aldrich, St. Louis, Missouri, USA $)$ at $65^{\circ} \mathrm{C}(0.25 \mathrm{U} / \mathrm{ml}$ final each time, first overnight, and then two treatments for 6 hours each). The reaction mixture was boiled for 15 minutes to inactivate residual enzyme and then dialyzed four times (molecular weight cut-off, $12-14 \mathrm{kDa}$ ) against $80 \mathrm{vol}$ of water containing $0.01 \%$ azide. The mixture was adjusted to $50 \mathrm{mM}$ Tris $\mathrm{HCl}, 100 \mathrm{mM} \mathrm{NaCl}, 2 \mathrm{mM} \mathrm{CaCl}_{2}, 2 \mathrm{mM}$ $\mathrm{MgCl}_{2}, 0.01 \%$ azide, $\mathrm{pH} 7.4$, and treated with Flavobacterium heparinum heparinase II (final, $0.075 \mathrm{U} / \mathrm{ml}$; SigmaAldrich) and Proteus vulgaris chondroitinase ABC (final, $0.015 \mathrm{U} / \mathrm{ml}$; Sigma-Aldrich) for 6 hours at $37^{\circ} \mathrm{C}$, and boiled for 15 minutes. The mixture was then treated twice with DNase $(75 \mathrm{U} / \mathrm{ml}$; Invitrogen Corp., San Diego, California, USA) and RNase $(0.01 \mathrm{mg} / \mathrm{ml}$; Invitrogen Corp.) for 2 hours at $37^{\circ} \mathrm{C}$, followed by one final proteinase $\mathrm{K}$ digestion, as described above. The mixture was then boiled for 15 minutes, dialyzed four times against water as described above, and lyophilized. The surviving large mucin fragments were then collected as a more than $200-\mathrm{kDa}$ void volume fraction by size exclusion chromatography (Sephacryl HR-S-200, $1.5 \mathrm{~cm} \times 95$ $\mathrm{cm}$; Pharmacia Biotech AB, Uppsala, Sweden) in isotonic buffer (50 mM Tris, $100 \mathrm{mM} \mathrm{NaCl}, 0.01 \% \mathrm{NaN}_{3}, \mathrm{pH}$ 7.4; flow rate $0.3 \mathrm{ml} / \mathrm{min}$, room temperature [RT]). The void volume fraction was dialyzed as described above, lyophilized, weighed, and stored at $-80^{\circ} \mathrm{C}$.

Mucin characterization. SDS-PAGE was performed using a 3\% stacking and a 4\% separating gel. Gels were stained by the periodic acid Schiff (PAS) method (61). Amino acid analysis was performed at the University of California, San Diego (UCSD) Core Facility by hydrolysis $6 \mathrm{M}$ $\mathrm{HCl}$ for 60 minutes at $160^{\circ} \mathrm{C}$ in sealed tubes, high-performance sodium cation exchange chromatography (Pickering Labs column) using a sodium citrate buffer system, postcolumn ninhydrin derivatization, and detec- 
tion peaks at $500-570 \mathrm{~nm}$, using norleucine as an internal standard. Monosaccharide analysis was performed by high-performance anion exchange chromatography with pulsed amperometric detection at the UCSD Glycotechnology Core Facility (http://grtc.ucsd.edu/glycocore.html). Sialic acid content was determined by reversed-phase HPLC analysis of 1,2-diamino-4,5-methylenedioxybenzene derivatives (62). Sulfate content was determined by IC analysis after pyrolysis at the UCSD Glycotechnology Core Facility. Selectin-binding activity was determined by ELISA, as previously described (35). Mucin preparations (500 ng/well) were coated onto ELISA plates overnight at $4^{\circ} \mathrm{C}$ in ELISA coating buffer (50 mM sodium carbonate/bicarbonate, $\mathrm{pH}$ 9.5). Soluble recombinant murine P-, L- or E-selectin/human IgG Fc chimera probes were precomplexed with goat antihuman IgG Ab (BioRad Laboratories Inc., Hercules, California, USA) in ELISA buffer (20 mM HEPES, $125 \mathrm{mM}$ $\mathrm{NaCl}, 2 \mathrm{mM} \mathrm{CaCl}$, $2 \mathrm{mM} \mathrm{MgCl}_{2}, 1 \% \mathrm{BSA}, 0.1 \% \mathrm{NaN}_{3}$, $\mathrm{pH}$ 7.4) for 1 hour at room temperature while rocking, and the mixture was exposed to the coated wells overnight at $4^{\circ} \mathrm{C}$. Following washing, binding was detected with pnp-phosphate substrate.

Coagulation assays. Various concentrations of mucin were added to citrated human plasma, which was then recalcified to test for contaminating TF using a coagulometer (Diagnostica Stago Inc., Parsippany, New Jersey, USA) that detects fibrin formation. A standard curve of added TF (Dade Innovin; Baxter Healthcare Corp., Deerfield, Illinois, USA) established that the assay sensitivity for a clotting response to TF was in the subnanogram range. Platelet aggregometry assays (Chrono-Log Corp., Havertown, Pennsylvania, USA) were performed on hirudinized platelet-rich plasma (PRP) using mucin and/or ADP, arachidonic acid, epinephrine, and collagen (Bio/Data Corp., Horsham, Pennsylvania, USA) (63).

Pyrogenicity assays. Mononuclear cells (MNCs) were isolated from pooled citrated mouse blood by isopycnic centrifugation through Ficoll-Paque research grade resolving medium (Pharmacia Biotech AB). MNCs $\left(2 \times 10^{6}\right.$ MNC/ml RPMI; Gibco 11875-093) were cultured with or without mucin $(2-500 \mu \mathrm{g} / \mathrm{m})$. After 20 hours of incubation, the supernatant was assayed for secreted IL- 6 by sandwich ELISA using a BD PharMingen kit (San Diego, California, USA). LPS (Sigma-Aldrich) was used as a reference standard and demonstrated the sensitivity of the assay to be approximately 0.001 endotoxin $\mathrm{U} / \mathrm{ml}$. Mucin was not positive in this assay and also did not inhibit LPS activity. Note that $100 \mu \mathrm{g}$ mucin and $2 \times 10^{6} \mathrm{MNCs} / \mathrm{ml}$ approximates relative concentrations expected in the in vivo studies.

In vivo studies. Lyophilized purified mucin was dissolved in tissue culture-grade PBS (Invitrogen Corp., Grand Island, New York, USA) and injected (50-400 $\mu \mathrm{g} / 100 \mu \mathrm{l})$ into the lateral tail vein of mice anesthetized with isoflurane (VEDCO Inc., St. Joseph, Missouri, USA). Heparin (Fujisawa Healthcare Inc., Deerfield, Illinois, USA) at 100 United States Pharmacopeia units per mouse (35) was injected 15 minutes before injec- tion of the mucin. Hirudin (Refludan; Aventis Pharmaceuticals Inc., Bridgewater, New Jersey, USA) at 50 $\mu \mathrm{g} /$ mouse was coinjected with the mucin. In some experiments murine thrombin (Sigma-Aldrich) was coinjected (0.001-0.05 U/mouse) with mucin.

Histology. Lung morphology was preserved in euthanized mice by intratracheal perfusion of a 1:1 vol/vol mixture of PBS and histological embedding medium (TissueTek OCT compound; Sakura Finetek, Torrance, California, USA) prior to opening the thoracic cavity. Acetone-fixed cryosections $(5 \mu \mathrm{m})$ were blocked in $10 \%$ rabbit serum, incubated with sheep anti-human fibrin(ogen) (BIODESIGN International Inc., Saco, Maine, USA) (PBS, $1 \% \mathrm{BSA}, 0.1 \% \mathrm{NaN}_{3}, 4^{\circ} \mathrm{C}$, overnight), FITC-conjugated rabbit anti-sheep IgG heavy \& light ( $\mathrm{H} \& \mathrm{~L})$ chains, purified rat anti-mouse CD41 (BD PharMingen), AffiniPure $\mathrm{Cy}^{3}$-conjugated donkey anti-rat $\mathrm{IgG}(\mathrm{H} \& \mathrm{~L})$ (Jackson ImmunoResearch Laboratories Inc., West Grove, Pennsylvania, USA), and mounted with Vectashield mounting medium containing DAPI (Vector Laboratories Inc., Burlingame, California, USA). Per tissue section, 20 random fields at $200 \times$ magnification were digitally captured and subjected to histographic quantification of immunofluorescently labeled (CD41-Cy3) platelet-positive pixels using Adobe Photoshop (64).

Whole-blood assays. Whole blood was collected directly by cardiac puncture in tubes containing Refludan (50 $\mu \mathrm{g} / \mathrm{ml}$, final). Fifty microliters of blood was stirred (approximately $1,000 \mathrm{rpm}, 37^{\circ} \mathrm{C}$ ) in siliconized cuvettes with magnetic stir bars (Crono-Log Corp.). Five microliters of diluted test compounds were added to the stirring blood and allowed to mix for 5 minutes. Reacted blood $(2 \mu \mathrm{l})$ was then diluted into $20 \mu \mathrm{l}$ HEPES-buffered saline containing biotin-conjugated rat anti-mouse P-selectin (CD62P) $(5 \mu \mathrm{g} / \mathrm{ml}$ final), FITC-conjugated rat anti-mouse CD41 (BD PharMingen) $(5 \mu \mathrm{g} / \mathrm{ml}$ final), and streptavidin-R-phycoerythrin (Jackson ImmunoResearch Laboratories Inc.) $(5 \mu \mathrm{g} / \mathrm{ml}$ final). Labeling steps were performed sequentially by static incubation (15 minutes, RT). Unfixed, diluted, labeled blood was immediately analyzed by flow cytometry and results analyzed with CellQuest software (Becton Dickinson Immunocytometry Systems, San Jose, California, USA). Platelets were gated by FITC fluorescence and the percentage of P-selectin-positive (CD62P-PE) cells determined. Baseline P-selectin expression obtained with PBS-treated controls was subtracted as background. Data was considered significant when baseline was less than $10 \%$ of CD 41 -FITC-gated platelets.

Statistical analysis. Data are expressed as mean plus or minus standard error of the mean. Comparisons used the Student's $t$ test.

\section{Results}

Purification of large-carcinoma mucin fragments. Early reports $(44,45)$ and some medical textbooks suggest that carcinoma mucins can initiate coagulation by direct activation of factor $\mathrm{X}$. Contamination by TF and/or other bioactive molecules, however, likely confounded 


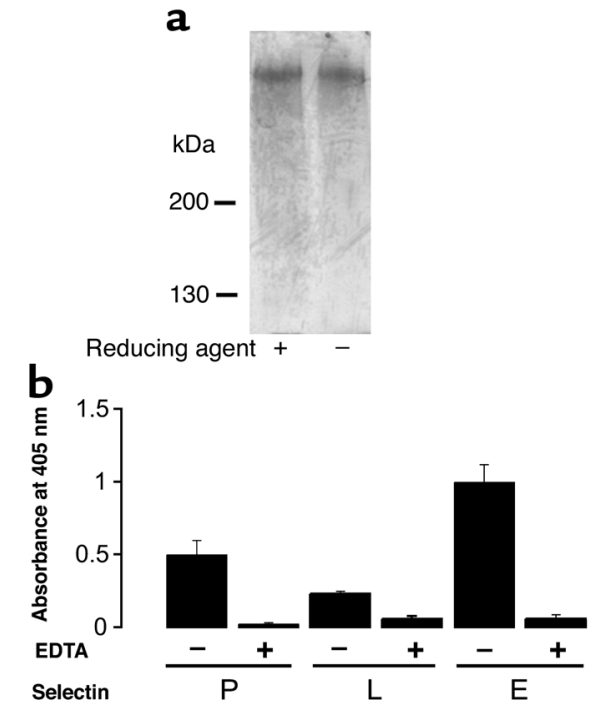

such studies (D. Le and S. Rapaport, unpublished observations). This is not surprising, since intact mucins are large complex molecules that can associate noncovalently with many tissue-derived lipids and proteins.

Proteolytic fragments of mucins are often released into the blood by tumor cells, and the heavily glycosylated domains of mucins are resistant to proteases because of shielding by the clustered O-glycans $(22-24,65)$. Taking advantage of the protease resistance, we developed a novel method for the purification of large mucin fragments from xenografted tumors, defined as greater than 200$\mathrm{kDa}$ domains sufficiently glycosylated as to be protected from the action of a broad-spectrum protease. The rationale for the overall approach was to eliminate all contaminating lipids and polypeptides, including procoagulant molecules such as phospholipids, platelet-activating factor, and TF. Also, the final product would be a

\section{Figure 1}

Purified mucin preparations are large and retain calcium-dependent binding sites for all three selectins. (a) A 4\% SDS-PAGE gel with detection of carbohydrates by PAS reagent. (b) ELISA in which immobilized mucin preparations are probed with recombinant soluble murine selectins. Values represent mean $\pm \mathrm{SEM} ; n=5$ individual mucin preparations. P, P-selectin; L, L-selectin; E, E-selectin.

mixture of proteolytic fragments of various mucin polypeptides, similar to that secreted by naturally occurring cancers. As described in Methods, the complete procedure involved extensive lipid extraction and broad-spectrum protease digestions followed by other enzyme treatments to remove large polyanionic molecules such as glycosaminoglycans and nucleic acids and eventual gel filtration to collect the surviving large mucin fragments.

Analysis of such material by gel electrophoresis followed by carbohydrate detection with the PAS stain revealed a high-molecular-weight band of more than $200 \mathrm{kDa}$ that entered into the 4\% SDS gel (Figure 1a). As shown in Table 1, the chemical composition of these purified carcinoma mucin preparations was as expected for mucins. The preparations were typically approximately $30 \%$ amino acid and approximately $60 \%$ carbohydrate by weight. Amino acid analysis revealed enrichment in serine, threonine, and proline, all characteristic of mucins. Monosaccharide compositional analysis also showed a profile characteristic of O-linked glycans, with a dominance of $\mathrm{N}$-acetylgalactosamine and the near absence of mannose. Sialic acids constituted approximately $15 \%$ of the total weight of carbohydrate, and sulfate contributed approximately $1.5 \%$ of the total weight. This analysis also showed the absence of potential contaminants such as extracellular matrix glycosaminoglycans (glucuronic acid and iduronic acid absent), nucleic acids (ribose and deoxyribose absent), and LPS (KDO and heptose absent).

Table 1

Chemical composition of purified carcinoma mucin preparations from LS180 xenograft tumors

\begin{tabular}{lcccc}
\cline { 2 - 2 } Amino acid & $(\mathrm{mol} / \mathbf{1 0 0}$ mol amino acid $)$ & Monosaccharide & (mol/mol GalNAc) & $(\mu \mathrm{g} / 100 \mu \mathrm{g}$ mucin $)$ \\
Asx & $2.4 \pm 0.3$ & Sia & $2.24 \pm 0.26$ & $13.01 \pm 0.99$ \\
Thr & $32.1 \pm 1.5$ & GalNAc & $1.00 \pm 0.00$ & $5.51 \pm 0.60$ \\
Ser & $12.2 \pm 0.9$ & GlcNAc & $1.57 \pm 0.19$ & $7.86 \pm 0.32$ \\
Glx & $4.3 \pm 0.3$ & Man & $0.21 \pm 0.10$ & $0.83 \pm 0.35$ \\
Pro & $17.8 \pm 1.1$ & Fuc & $1.02 \pm 0.13$ & $4.51 \pm 0.22$ \\
Gly & $5.2 \pm 0.6$ & Gal & $1.96 \pm 0.21$ & $9.15 \pm 0.17$ \\
Ala & $6.6 \pm 0.8$ & Glc & $0.17 \pm 0.07$ & $0.63 \pm 0.39$ \\
Val & $4.1 \pm 0.4$ & Xyl & N.D. & N.D. \\
lle & $3.3 \pm 0.1$ & GalA & N.D. & \\
Leu & $2.2 \pm 0.6$ & GlcA & N.D. & N.D. \\
Tyr & $1.0 \pm 0.6$ & IdoA & N.D. \\
Phe & $1.1 \pm 0.3$ & Rib & N.D. \\
His & $3.2 \pm 0.9$ & dRib & N.D.
\end{tabular}

Several batches of purified mucin were analyzed for chemical composition. Values represent the mean \pm SEM of eight individual preparations. N.D., not detected; Sia, sialic acid; GalNAc, N-acetylgalactosamine; GlcNAc, N-acetylglucosamine; Man, mannose; Fuc, fucose; Gal, galactose; Glc, glucose; Xyl, xylose; GalA, galacturonic acid; GlcA, glucuronic acid; IdoA, iduronic acid; Rib, ribose; dRib, deoxyribose; KDO, 2-keto,3-deoxyoctulosonic acid. 
a
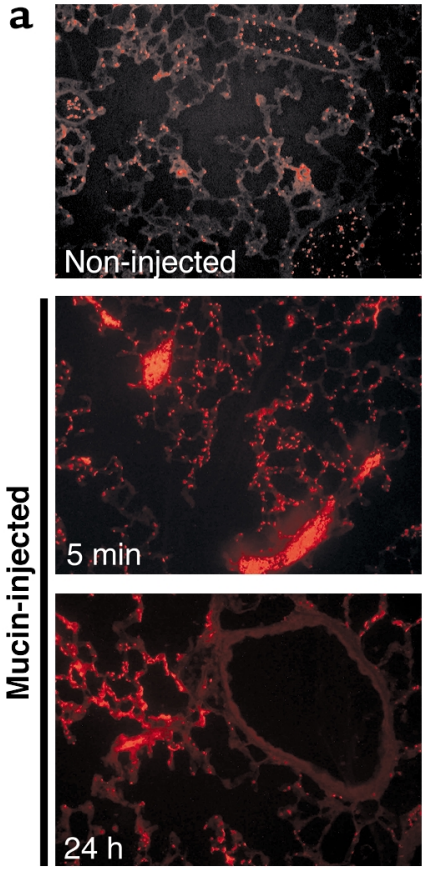
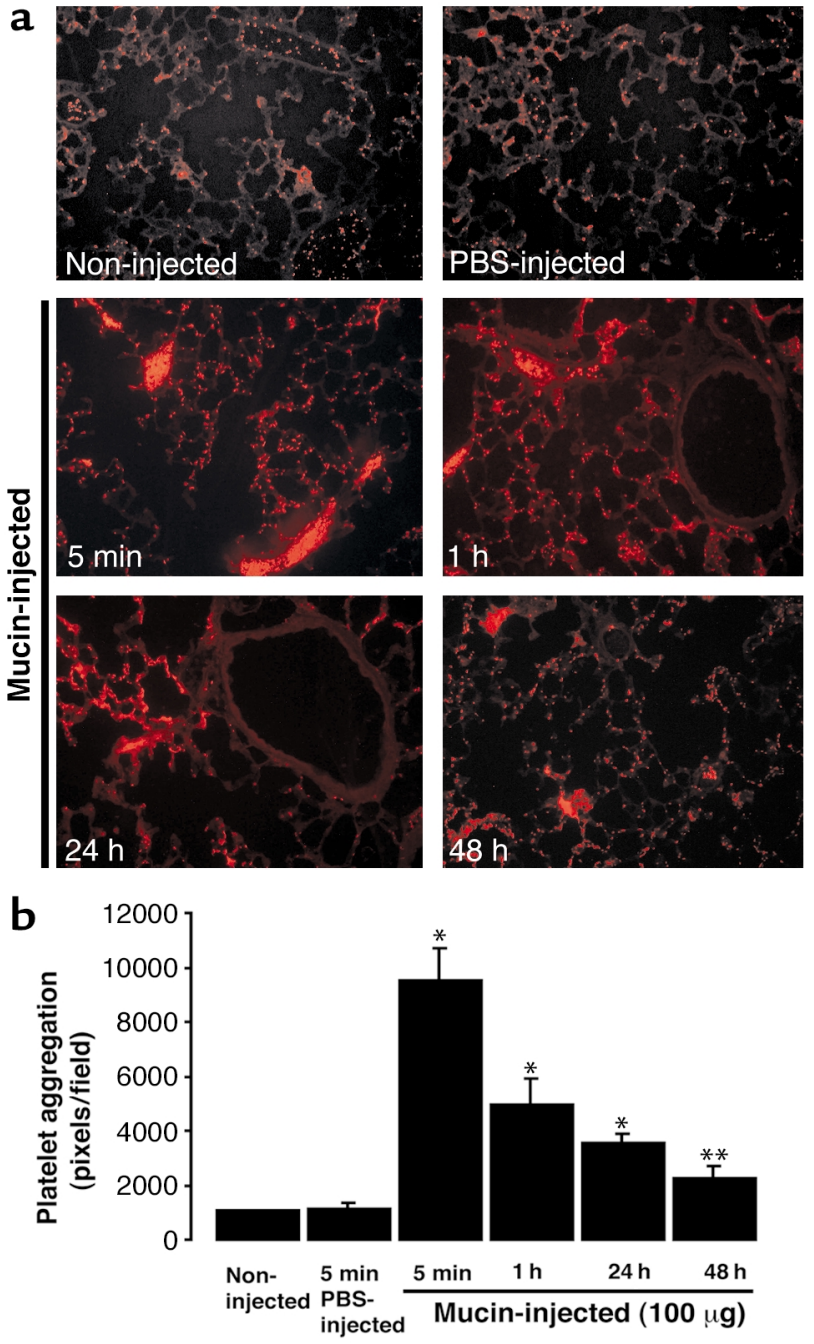

The mucin preparations are recognized by selectins in a calcium-dependent manner. Intact mucins isolated from LS180 adenocarcinoma xenografts are known to be recognized by all three selectins in a calcium-dependent manner (33). To determine if the mucin preparations from tumors retained functional binding sites for selectins, we performed ELISAs that quantified the binding of recombinant soluble mouse selectin Ig-Fc chimeras to the mucin preparations on a stationary phase. As shown in Figure 1b, the mucins do retain calciumdependent binding sites for all three selectins. In addition, we found that the $\mathrm{IC}_{50}$ values of mucin preparations (concentration giving 50\% inhibition) of P-selectin binding to a stationary phase of Sialyl-Lewis $\mathrm{X}$ were consistent from batch to batch (data not shown). Thus, the novel method of purification described herein reproducibly yields high-molecular-weight mucin preparations that are recognized by all three selectins in a calcium-dependent manner.

The mucin preparations do not activate fluid-phase coagulation pathways. The mucin preparations failed to initiate clotting in a modified prothrombin assay using normal human plasma, indicating the absence of con-

\section{Figure 2}

Time-course of platelet-rich microthrombi in wild-type mouse lungs following a single injection of mucin. (a) Typical representations of in vivo platelet-rich microthrombi in lung sections observed using fluorescently labeled Ab against the platelet marker, CD41. (b) Quantitative histologic determination, quantitating average number of CD41-positive pixels per 200x view field (mean value of 20 random fields). Each bar represents the mean \pm SEM. ${ }^{*} P<0.001 \mathrm{com}$ pared with PBS-injected mice, $n=7 ;{ }^{*} P<0.05, n=5$.

taminating TF (data not shown; assay sensitivity was in the subnanogram range). The mucin preparations also did not affect the activated partial thromboplastin and dilute Russel's viper venom tests on normal plasma, ruling out any trace lipids that might influence coagulation (data not shown). Thus, the mucin preparations are free of TF and are not anti- or procoagulant in standard in vitro assays.

The mucin preparations are not pyrogenic. Although sterile procedures were followed throughout, contamination could have arisen from the enzyme preparations used or from unexpected sources. As expected for large polyanionic molecules, these mucin fragments gave a weak false-positive assay in the standard Limulus lysate assay for endotoxin. Individual batches of mucin were therefore screened for pyrogenic activity using a more sensitive and specific mouse MNC-based assay in which samples or controls are added to the culture medium, with detection of secreted IL- 620 hours later by sandwich ELISA (66). From negative results in these studies (data not shown), we concluded that mucin preparations are not pyrogenic.

The mucin preparations do not activate platelets in vitro. We have shown that carcinoma mucin preparations alone do not directly activate platelets in vitro $(33,34)$. In the presence of subthreshold levels of thrombin, however, mucins did enhance platelet aggregation in vitro. This effect of mucin was calcium dependent and could be blocked by P-selectin-blocking Ab's and also did not occur if platelets from P-selectin ${ }^{-/-}$mice were used (34). These findings were obtained using washed platelets suspended in isotonic buffer. To study platelets under somewhat more physiological conditions we used stirred PRP anticoagulated with hirudin (Refludan), a leech-derived irreversible and instantaneous thrombin inhibitor that is currently in clinical use. Under these conditions, the mucin preparations again had no effect on platelet aggregation in the absence of thrombin. Moreover, they did not enhance aggregation in response to subthreshold levels of other platelet agonists, such as ADP, epinephrine, and collagen (data not shown). Thus, the carcinoma mucin preparations do not activate platelets directly in the absence of active thrombin.

Intravenous injection of mucin preparations into mice generates platelet-rich microthrombi. In contrast to the lack of bioactivity in all the above in vitro assays, the mucin preparations rapidly generated platelet-rich intravascular microthrombi (detected with anti-CD41, a platelet 
a
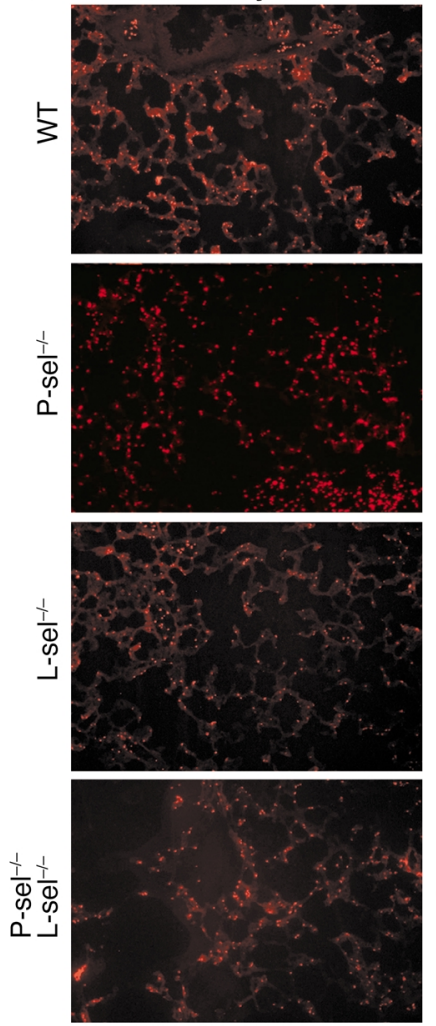

b

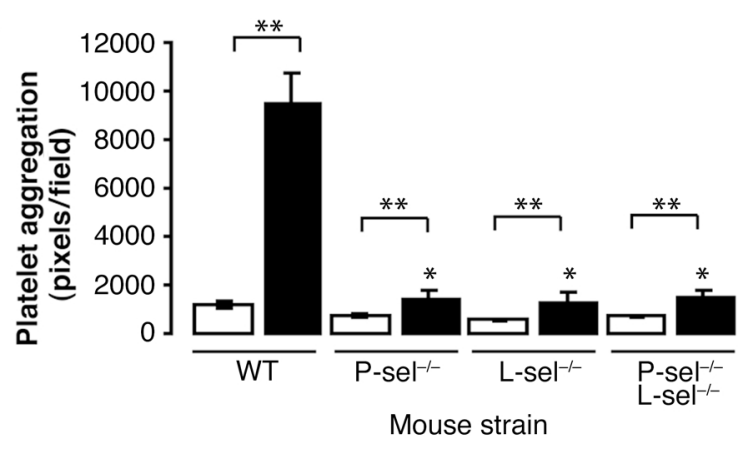

marker) when they were injected intravenously into mice. These microthrombi were found in several tissues including liver sinusoids, renal glomeruli, choroid plexus of brain, and lung. Since the small blood vessels of the lung were the most consistent sites of platelet aggregation, we quantified the extent of platelet accumulation in lung sections by taking digitized photographs and determining the average number of CD41-positive pixels per view field (Figure 2). The platelet-rich microthrombi were observed within 5 minutes after a single injection of the mucin preparation, and nearly half were resolved after 1 hour. Some microthrombi persisted, however, and did not fully resolve until after 48 hours. The microthrombi could be seen with as little as $50 \mu$ g of mucin injected per mouse (data not shown), which equates with a plasma concentration of approximately $50 \mu \mathrm{g} / \mathrm{ml}$ (assuming a mouse plasma volume of approximately $1 \mathrm{ml}$ ). Notably, some cancer patients can have circulating mucin concentrations as high as approximately $100 \mu \mathrm{g} / \mathrm{ml}$ (as deter-

\section{Figure 3}

Selectin deficiency attenuates platelet-rich microthrombus formation following mucin injection. (a) Typical representations of platelet-rich microthrombi in lung sections observed as in Figure 2, at 5 minutes after injection. (b) Quantitative determination of CD41 in lung sections as in Figure 2. Values shown represent the mean \pm SEM. ${ }^{*} P<0.001 \mathrm{com}$ pared with mucin-injected wild-type mice, $n=7 ;{ }^{*} P<0.05, n=7$. White bars, PBS-injected mice; black bars, mucin-injected mice.

mined by the CA 19-9 immunoassay that detects sialyl Lewis A epitopes carried on mucins) $(67,68)$.

In vivo effects of mucin are dependent on P-selectin and $L$-selectin. To determine if selectins play a role in the in vivo response to injected mucins, we repeated mucin injections in wild-type versus selectin-deficient mice. As shown in Figure 3, nearly all the alveolar capillaries in wild-type mice were occupied by small platelet aggregates. Large aggregates also occupied some medium-sized veins. In contrast, mucin injection into P- or L-selectin-deficient mice yielded scant small aggregations that were barely above background levels. Mice with a combined deficiency of both selectins did not show additional suppression beyond that seen with either single selectin deficiency. Thus, the mucin effect must operate via a linear pathway involving both $\mathrm{P}$ - and L-selectin. We did not explore the role of E-selectin in this system because it is not normally present on endothelium, but must be transcriptionally activated by specific stimuli (69).

In addition to its anticoagulant action, heparin is also known to block P- and L-selectin interactions (57). Indeed, recent work suggests that most or all of the anti-inflammatory effects of heparin can be explained by selectin blockade (59). In keeping with this, we found that heparin injection 15 minutes before mucin injection significantly reduced the platelet-rich microthrombi in wild-type mice (Figure 4a). This protective role of heparin in wild-type mice, however, could also be due to its antithrombin/anticoagulant activity. Thus, we next investigated the role of thrombin, attempting to disentangle the fluid-phase coagulation component from the selectin component.

Initial aggregation of platelets is independent of thrombin activity. Our original hypothesis (based on in vitro data) was that tumor-derived TF might generate low levels of thrombin, which would then generate a circulating population of platelets with exposed P-selectin. Mucins would then aggregate those platelets by cross-linking P-selectin, perhaps starting a positive feedback loop and recruiting additional platelets to the aggregates. To examine this possibility, subthreshold levels of thrombin were coinjected with mucin. The effects of thrombin, however, were simply additive rather than synergistic (Figure 4b). Surprisingly, platelet aggregation remained unchanged, even when we inhibited in vivo thrombin activity by coinjecting hirudin along with mucin (see Figure 4a). To verify that hirudin was actually working in vivo we studied the platelet-rich microthrombi in more detail, specifically focusing on fibrin (an indicator of 


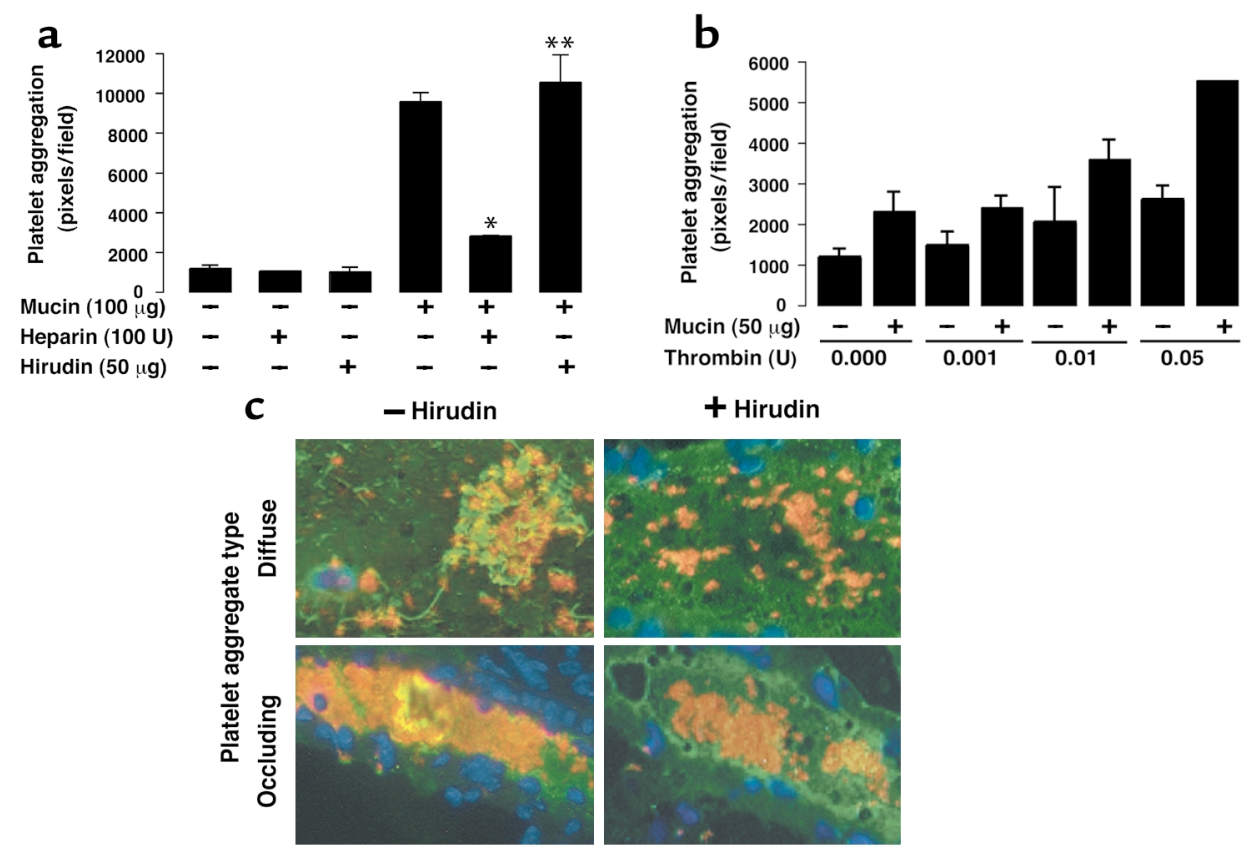

\section{Figure 4}

Relative importance of fluid-phase coagulation versus selectins in mucin-induced microthrombus formation. (a and b) Quantitative determination of CD41 in lung sections as in Figure 2, at 5 minutes after injection. (a) Ability of heparin or hirudin to block the effects of mucin in wild-type mice. ${ }^{*} P<0.05$ compared with mucin alone, $n=5$; ${ }^{*} P<0.001$ compared with PBS-injected mice, $n=5$. (b) Effects of coinjecting subthreshold levels of thrombin with mucin. (c) Three-color immunofluorescence microscopic analysis of platelet-rich microthrombi in wildtype mice injected with mucin (with or without hirudin) to demonstrate fibrin, an indicator of thrombin activity. Bright green indicates deposited fibrin; pale green indicates autofluorescence of luminal surface of longitudinally sectioned venule; red indicates platelets; and blue indicates nuclei. Examples of occluding microthrombi and diffuse platelet aggregates are shown.

thrombin activity) and on platelets and leukocytes (Figure 4c). While hirudin at the same dose did prevent fibrin deposition within and around all microthrombi, the aggregation of platelets was unaffected. The morphology of the platelet aggregates in the presence of hirudin did look a little different, presumably because the fibrin strands were not available to consolidate the aggregates.

Because mucin did cause some low-grade platelet aggregation in selectin-deficient mice (see Figure 3), we next studied the effects of heparin and hirudin in these mice. Hirudin, with or without added heparin, did not affect the residual low-grade platelet aggrega- tion in $\mathrm{P}-\mathrm{sel}^{-/-}, \mathrm{L}_{-} \mathrm{sel}^{-/-}$, or P-sel $\mathrm{P}^{-/-} / \mathrm{L}-\mathrm{sel}^{-/-}$mice (data not shown). Taken together, these data indicate that thrombin is not a proximal component in the pathway of mucin-induced platelet aggregation in this in vivo model. Rather, fibrin deposition (an index of thrombin activity) appears to be a secondary process. Mucins activate platelets via lenkocyte $L$-selectin in whole blood. Because mucins do not activate platelets in buffer (34) or in PRP (data not shown), and the in vivo effects of mucin appeared to be thrombin independent and L-selectin dependent, we hypothesized that leukocytes were involved in activating platelets. To test this in vitro, stirred

\section{Table 2}

Microangiopathic characteristics in mice bearing mucinous tumors

$\begin{array}{lccc} & \text { Normal } & \text { Tumor-bearing } & P \text { value } \\ \text { rbc count }\left(10^{6} / \mu \mathrm{l}\right) & 10.9 \pm 0.3 & 8.4 \pm 0.6 & 0.01 \\ \text { Hemoglobin }(\mathrm{g} / \mathrm{dl}) & 14.0 \pm 0.3 & 11.2 \pm 0.6 & 0.01 \\ \text { Polychromatophilic macrocytes/1,000 rbc's } & 5.8 \pm 0.1 & 24 \pm 4 & 0.06 \\ \text { Schistocytes (fragmented rbc's)/1,000 rbc's } & 0.5 \pm 0.2 & 2.2 \pm 0.5 & 0.002 \\ \text { Serum lactate dehydrogenase }(\mathrm{IU} / \mathrm{l}) & 701 \pm 816 & 2,829 \pm 816 & 0.007 \\ \text { Total bilirubin }(\mathrm{mg} / \mathrm{dl})_{\text {Platelet aggregates }\left(\text { pixels/field) }^{\mathrm{A}}\right.} & 0.25 \pm 0.057 & 0.39 \pm 0.036 & 0.04 \\ & 917.5 \pm 175 & 3,163.4 \pm 727 & 0.005\end{array}$

Mice carrying xenografts of LS180 human cells were screened for clinical signs of microangiopathy and compared with non-tumor-bearing littermates. Values represent the mean \pm SEM of values obtained in multiple cohorts of wild-type $(n=5-15)$ and tumor-bearing $(n=12-38)$ mice. Statistical significance is shown using the Student's $t$ test. APlatelet aggregation was determined by histologic evaluation of lung sections as in Figure 2 . Some amount of background aggregation is observed in wild-type mice due to the autopsy procedure. 


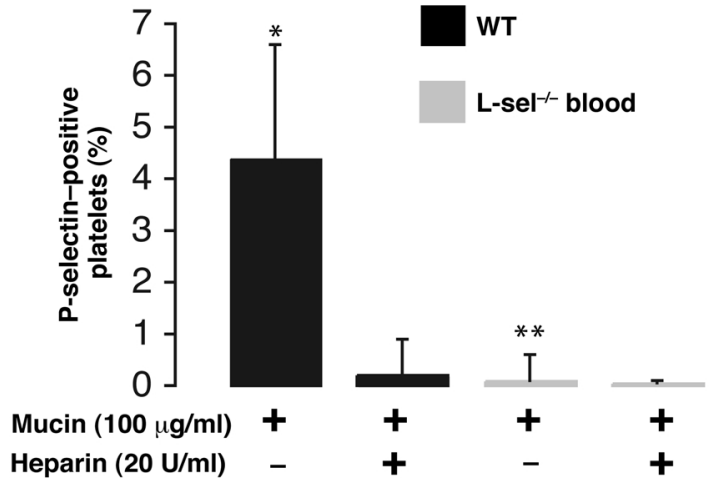

Figure 5

In vitro demonstration of the essential role of leukocyte L-selectin in mediating platelet activation. Flow-cytometric quantification of platelet activation based on P-selectin expression in stirred, hirudinanticoagulated whole blood. Black bars, wild-type mouse blood; gray bars, L-sel-/- mouse blood. Values represent the mean \pm SEM $(n=3) ;{ }^{*} P=0.007$ relative to PBS-treated controls; ${ }^{*} P=0.003$ relative to mucin-treated wild-type blood.

mouse whole blood, anticoagulated with hirudin to inhibit thrombin was incubated at $37^{\circ} \mathrm{C}$ with various test compounds for 5 minutes, followed by flow-cytometric detection and quantification of activated platelets by testing expression of P-selectin (Figure 5). A low but significant degree of P-selectin expression was observed, consistent with leukocyte-mediated platelet activation rather than direct stimulation of platelets (70). While the mucin preparations did activate platelets in wild-type whole blood, they were inactive in whole blood from L-sel-/mice. Furthermore, heparin completely blocked the effect of mucin in wild-type blood. Since hirudin is already used in a saturating amount, heparin is likely acting through its role as an inhibitor of P- and/or L-selectin interactions, rather than as a thrombin inhibitor. The final concentration of mucin used in this in vitro system was about the same as that anticipated in the in vivo experiments. From these results we conclude that mucins require leukocyte L-selectin in order to activate platelets, causing them to express P-selectin (see discussion below).

Mice bearing mucin-producing tumors show evidence for Troussean syndrome. Some of the mice carrying xenografts from which mucin was extracted for the current studies did show clinical signs of microangiopathic hemolytic anemia (Table 2). They were anemic, had moderate amounts of circulating damaged red blood cells (schistocytes), and had high numbers of young red cells (polychromatophilic macrocytes, consistent with hemolysis and compensatory enhanced erythropoiesis. Serum chemistry revealed increased LDH and bilirubin, consistent with a hemolytic process. Histologic analysis of lungs revealed mild to severe disseminated plateletrich microthrombi in some of the mice. Many further studies will, of course, be needed to prove that the corroborative findings in these animals are indeed related to circulating tumor mucins and to extend these observations to humans with cancer.

\section{Discussion}

We have shown that carcinoma mucins alone can activate platelets and generate the classic microangiopathic form of Trousseau syndrome in intact mice. This provides a rational explanation for the previously mysterious association of Trousseau syndrome with mucin-producing carcinomas. Our original hypothesis that TF was required for mucin to act as a template and aggregate activated platelets through P-selectin was not substantiated, and we were surprised to find that L-selectin was also essential. L-selectin signaling normally activates leukocytes, presumably because the cell must attain new properties in order to extravasate effectively at sites of endogenous L-selectin ligands. Thus, mucins may initially ligate L-selectin, generating various signals that eventually result in systemic activation of platelets, aggregation, and embolization. Subsequent propagation of these lesions could then arise through P-selectin-based interactions, as well as secondary activation of coagulation.

Although L-selectin is an adhesion molecule, it also has signaling functions that cause cellular activation (71-80). Thus, we propose a model for L-selectin-mediated activation of platelets that relies on a leukocyteplatelet cross-talk mechanism rather than on the fluid phase coagulation system (Figure 6). This P- and L-selectin-dependent mechanism leads to the activation and aggregation of platelets via a pathway that is thrombin independent. L-selectin signaling by the mucin activates leukocytes, which then activate platelets through an as yet unknown mediator. Given that the effects are seen within 5 minutes both in vitro and in vivo, the latter must be a rapidly generated molecule.

Our results also emphasize a critical role for P-selectin, which could be required for platelet aggregation brought about by mucin cross-linking of P-selectin-expressing

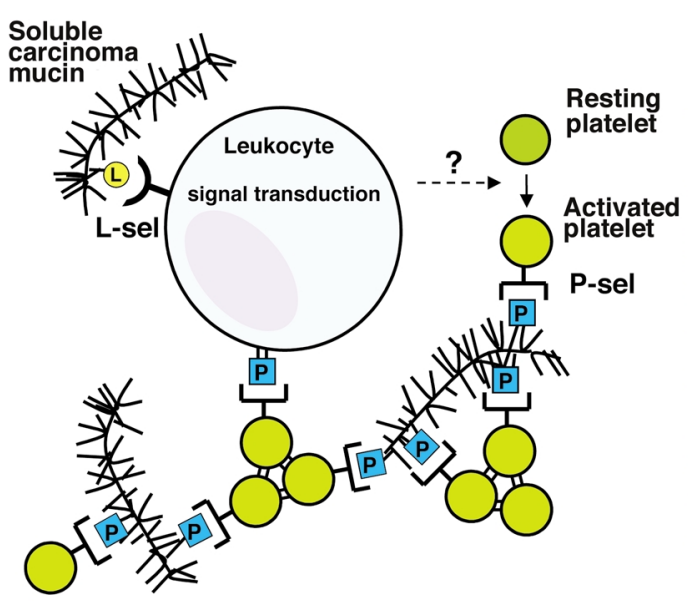

Figure 6

Proposed model for L- and P-selectin-mediated mucin-induced activation and aggregation of platelets. Yellow circle labeled with $L$ indicates L-selectin ligands on mucins. Blue boxes labeled with P indicate P-selectin ligands, including carcinoma mucins and PSGL-1 expressed on leukocytes. L-sel, L-selectin; P-sel, P-selectin. 
platelets (Figure 6). Alternatively (or in addition), P-selectin interaction with its endogenous ligand, leukocyte P-selectin glycoprotein ligand-1 (PSGL-1), might induce cross-signaling in leukocytes (Figure 6). PSGL-1 signaling activates integrins (CD11b/CD18) on neutrophils $(81,82)$, transcellular metabolism of arachidonic acid (83), and thromboxane $A_{2}$ generation (84). PSGL-1 signaling also upregulates TF generation by monocytes (85-88). Finally, a role for P-selectin in interacting with sulfatide glycolipid ligands expressed on platelets and red blood cells was recently reported (89). Thus, the initial activation and aggregation of platelets caused by mucins by L- and P-selectin could, in turn, generate secondary responses, namely, activation of coagulation and recruitment of additional platelets as well as other leukocytes and red blood cells, generating the more complete lesions.

Our data indicate that activation of coagulation is not a proximal component in carcinoma mucin-generated platelet-rich microthrombi. Rather, the phenomenon is primarily mediated through $\mathrm{P}$ - and L-selectins. These findings are in agreement with the clinical observation that while heparin (which is known to also inhibit P- and L-selectin interactions) is the drug of choice for managing patients with Trousseau syndrome, other therapeutic strategies that attenuate the fluid-phase coagulation cascade are often ineffective $(2-10)$. Of course, we do not claim that mucins alone are responsible for all forms of Trousseau syndrome, according to the broader definition. Indeed, it is likely that tumor-derived TF as well as other procoagulants are involved in the broad spectrum of hypercoagulable states seen in cancer patients. It is also possible that such additional factors could directly associate with the large negatively charged mucins or mucin fragments, thereby potentiating their multiple effects. Additional studies are also required to determine whether specific mucin polyptides are the carriers of the critical selectin ligands. The possibility of using methods of selectin inhibition other than heparin for human cases associated with mucin-producing carcinomas should also be explored.

\section{Acknowledgments}

We gratefully acknowledge Q. Chen, D. Ditto, L. Kim, D. Matriano, and S. Smith for excellent technical assistance and for useful discussions. This work was supported by grant R01 CA38701 from the United States Public Health Service.

1. Trousseau, A. 1868. Lectures on clinical medicine, delivered at the Hotel-Dieu, Paris. P.V. Bazire, editor and translator. The New Sydenham Society Publications. London, United Kingdom. 55:281-332.

2. Samuels, M.A., King, M.E., and Balis, U. 2002. Case records of the Massachusetts General Hospital. Weekly clinicopathological exercises. Case 31-2002. A 61-year-old man with headache and multiple infarcts. N. Engl. J. Med. 347:1187-1194.

3. Sack, G.H., Jr., Levin, J., and Bell, W.R. 1977. Trousseau's syndrome and other manifestations of chronic disseminated coagulopathy in patients with neoplasms: clinical, pathophysiologic, and therapeutic features. Medicine (Baltimore). 56:1-37.

4. Callander, N., and Rapaport, S.I. 1993. Trousseau's syndrome. West. J. Med. 158:364-371.
5. Rigdon, E.E. 2000. Trousseau's syndrome and acute arterial thrombosis. Cardiovasc. Surg. 8:214-218.

6. Arkel, Y.S. 2000. Thrombosis and cancer. Semin. Oncol. 27:362-374.

7. Rickles, F.R., and Falanga, A. 2001. Molecular basis for the relationship between thrombosis and cancer. Thromb. Res. 102:V215-V224.

8. Gale, A.J., and Gordon, S.G. 2001. Update on tumor cell procoagulant factors. Acta Haematol. 106:25-32.

9. Lee, A. 2002. Cancer and thromboembolic disease: pathogenic mechanisms. Cancer Treat. Rev. 28:137-140.

10. Lip, G.Y., Chin, B.S., and Blann, A.D. 2002. Cancer and the prothrombotic state. Lancet Oncol. 3:27-34.

11. Gordon, S.G. 1992. Cancer cell procoagulants and their implications. Hematol. Oncol. Clin. North Am. 6:1359-1374.

12. Bani, M.R., et al. 1992. Blood coagulation changes in nude mice bearing human colon carcinomas. Int. J. Cancer. 50:75-79.

13. Mielicki, W.P., Tenderenda, M., Rutkowski, P., and Chojnowski, K. 1999. Activation of blood coagulation and the activity of cancer procoagulant (EC 3.4.22.26) in breast cancer patients. Cancer Lett. 146:61-66.

14. Callander, N.S., Varki, N., and Rao, L.V. 1992. Immunohistochemical identification of tissue factor in solid tumors. Cancer. 70:1194-1201.

15. Zacharski, L.R., Schned, A.R., and Sorenson, G.D. 1983. Occurrence of fibrin and tissue factor antigen in human small cell carcinoma of the lung. Cancer Res. 43:3963-3968.

16. Kakkar, A.K., DeRuvo, N., Chinswangwatanakul, V., Tebbutt, S., and Williamson, R.C. 1995. Extrinsic-pathway activation in cancer with high factor VIIa and tissue factor. Lancet. 346:1004-1005.

17. Bell, W.R., Starksen, N.F., Tong, S., and Porterfield, J.K. 1985. Trousseau's syndrome. Devastating coagulopathy in the absence of heparin. Am. J. Med. 79:423-430.

18. Krauth, D., Holden, A., Knapic, N., Liepman, M., and Ansell, J. 1987. Safety and efficacy of long-term oral anticoagulation in cancer patients. Cancer. 59:983-985.

19. Walsh-McMonagle, D., and Green, D. 1997. Low-molecular-weight heparin in the management of Trousseau's syndrome. Cancer. 80:649-655.

20. Meyer, G., et al. 2002. Comparison of low-molecular-weight heparin and warfarin for the secondary prevention of venous thromboembolism in patients with cancer: a randomized controlled study. Arch. Intern. Med. 162:1729-1735.

21. Levine, M. 2002. Managing thromboembolic disease in the cancer patient: efficacy and safety of antithrombotic treatment options in patients with cancer. Cancer Treat. Rev. 28:145-149.

22. Carraway, K.L., Fregien, N., Carraway, K.L., III, and Carraway, C.A. 1992. Tumor sialomucin complexes as tumor antigens and modulators of cellular interactions and proliferation. J. Cell Sci. 103:299-307.

23. Kim, Y.S., Gum, J., and Brockhause, I. 1996. Mucin glycoproteins in neoplasia. Glycoconj. J. 13:693-707.

24. Perez-Vilar, J., and Hill, R.L. 1999. The structure and assembly of secreted mucins. J. Biol. Chem. 274:31751-31754.

25. Hanisch, F.G., and Muller, S. 2000. MUC1: the polymorphic appearance of a human mucin. Glycobiology. 10:439-449.

26. Fukuda, M., and Tsuboi, S.T. 1999. Mucin-type O-glycans and leukosialin. Biochim. Biophys. Acta. 1455:205-217.

27. Nakamori, S., Ota, D.M., Cleary, K.R., Shirotani, K., and Irimura, T. 1994. MUC1 mucin expression as a marker of progression and metastasis of human colorectal carcinoma. Gastroenterology. 106:353-361.

28. Perrais, M., Pigny, P., Copin, M.C., Aubert, J.P., and Van, S.I. 2002. Induction of MUC2 and MUC5AC mucins by factors of the epidermal growth factor (EGF) family is mediated by EGF receptor/Ras/Raf/extracellular signal-regulated kinase cascade and Sp1. J. Biol. Chem. 277:32258-32267.

29. Yin, B.W., Dnistrian, T.A., and Lloyd, K.O. 2002. Ovarian cancer antigen CA125 is encoded by the MUC16 mucin gene. Int. J. Cancer. 98:737-740.

30. McCool, D.J., Forstner, J.F., and Forstner, G.G. 1995. Regulated and unregulated pathways for MUC2 mucin secretion in human colonic LS180 adenocarcinoma cells are distinct. Biochem. J. 312:125-133.

31. Enss, M.L., et al. 2000. Proinflammatory cytokines trigger MUC gene expression and mucin release in the intestinal cancer cell line LS180. Inflamm. Res. 49:162-169.

32. Komatsu, M., Arango, M.E., and Carraway, K.L. 2002. Synthesis and secretion of Muc4/sialomucin complex: implication of intracellular proteolysis. Biochem. J. 368:41-48.

33. Kim, Y J., Borsig, L., Han, H.L., Varki, N.M., and Varki, A. 1999. Distinct selectin ligands on colon carcinoma mucins can mediate pathological interactions among platelets, leukocytes, and endothelium. Am. J. Pathol. 155:461-472.

34. Kim, Y.J., Borsig, L., Varki, N.M., and Varki, A. 1998. P-selectin deficiency attenuates tumor growth and metastasis. Proc. Natl. Acad. Sci. U. S. A. 95:9325-9330.

35. Borsig, L., et al. 2001. Heparin and cancer revisited: Mechanistic connections involving platelets, P-selectin, carcinoma mucins, and tumor metastasis. Proc. Natl. Acad. Sci. U. S. A. 98:3352-3357.

36. Borsig, L., Wong, R., Hynes, R.O., Varki, N.M., and Varki, A. 2002. Synergistic effects of L- and P-selectin in facilitating tumor metastasis can 
involve non-mucin ligands and implicate leukocytes as enhancers of metastasis. Proc. Natl. Acad. Sci. U. S. A. 99:2193-2198.

37. Singhal, A.K. et al. 1990. Profiles of Lewisx-containing glycoproteins and glycolipids in sera of patients with adenocarcinoma. Cancer Res. 50:1375-1380.

38. Kawa, S., et al. 1991. Preparation of pancreatic cancer-associated mucin expressing CA19-9, CA50, Span-1, sialyl SSEA-1, and Dupan-2. Scand. J. Gastroenterol. 26:981-992.

39. Hanski, C., et al. 1995. Characterization of the major sialyl-Lex-positive mucins present in colon, colon carcinoma, and sera of patients with colorectal cancer. Cancer Res. 55:928-933.

40. Zhang, K., Baeckström, D., Brevinge, H., and Hansson, G.C. 1996. Secreted MUC1 mucins lacking their cytoplasmic part and carrying sialyl-Lewis $\mathrm{a}$ and $\mathrm{x}$ epitopes from a tumor cell line and sera of colon carcinoma patients can inhibit HL-60 leukocyte adhesion to E-selectin-expressing endothelial cells. J. Cell Biochem. 60:538-549.

41. Yiannakou, J.Y., Newland, P., Calder, F., Kingsnorth, A.N., and Rhodes, J.M. 1997. Prospective study of CAM 17.1/WGA mucin assay for serological diagnosis of pancreatic cancer. Lancet. 349:389-392.

42. Rhodes, J.M. 1999. Usefulness of novel tumour markers. Ann. Oncol. 4(Suppl. 10):118-121.

43. Yin, B.W., and Lloyd, K.O. 2001. Molecular cloning of the CA125 ovarian cancer antigen: identification as a new mucin, MUC16. J. Biol. Chem. 276:27371-27375.

44. Pineo, G.F., Regoeczi, E., Hatton, M.W., and Brain, M.C. 1973. The activation of coagulation by extracts of mucus: a possible pathway of intravascular coagulation accompanying adenocarcinomas. J. Lab. Clin. Med. 82:255-266.

45. Pineo, G.F., et al. 1974. Tumors, mucus production, and hypercoagulability. Ann. N. Y. Acad. Sci. 230:262-270.

46. Hemmerich, S., and Rosen, S.D. 2000. Carbohydrate sulfotransferases in lymphocyte homing. Glycobiology. 10:849-856.

47. Furie, B., Furie, B.C., and Flaumenhaf, R. 2001. A journey with platelet P-selectin: the molecular basis of granule secretion, signalling and cell adhesion. Thromb. Haemost. 86:214-221.

48. Tsuboi, S., and Fukuda, M. 2001. Roles of O-linked oligosaccharides in immune responses. Bioessays. 23:46-53.

49. McEver, R.P. 2002. Selectins: lectins that initiate cell adhesion under flow. Curr. Opin. Cell Biol. 14:581-586.

50. Varki, A. 1994. Selectin ligands. Proc. Natl. Acad. Sci. U. S. A. 91:7390-7397.

51. Kansas, G.S. 1996. Selectins and their ligands: current concepts and controversies. Blood. 88:3259-3287.

52. Epperson, T.R., Patel, K.D., McEver, R.P., and Cummings, R.D. 2000. Noncovalent association of P-selectin glycoprotein ligand-1 and minimal determinants for binding to P-selectin. J. Biol. Chem. 275:7839-7853.

53. Leppänen, A., Penttilä, L., Renkonen, O., McEver, R.P., and Cummings, R.D. 2002. Glycosulfopeptides with O-glycans containing sialylated and polyfucosylated polylactosamine bind with low affinity to P-selectin. J. Biol. Chem. 277:39749-39759.

54. Nelson, R.M., et al. 1993. Heparin oligosaccharides bind L- and P-selectin and inhibit acute inflammation. Blood. 82:3253-3258.

55. Norgard-Sumnicht, K.E., Varki, N.M., and Varki, A. 1993. Calciumdependent heparin-like ligands for $\mathrm{L}$-selectin in nonlymphoid endothelial cells. Science. 261:480-483.

56. Norgard-Sumnicht, K.E., and Varki, A. 1995. Endothelial heparan sulfate proteoglycans that bind to L-selectin have glucosamine residues with unsubstituted amino groups. J. Biol. Chem. 270:12012-12024.

57. Koenig, A., Norgard-Sumnicht, K.E., Linhardt, R., and Varki, A 1998. Differential interactions of heparin and heparan sulfate glycosaminoglycans with the selectins. Implications for the use of unfractionated and low molecular weight heparins as therapeutic agents. J. Clin. Invest. 101:877-889.

58. Xie, X., et al. 2000. Inhibition of selectin-mediated cell adhesion and prevention of acute inflammation by nonanticoagulant sulfated saccharides. Studies with carboxyl-reduced and sulfated heparin and with trestatin A sulfate. J. Biol. Chem. 275:34818-34825.

59. Wang, L., Brown, J.R., Varki, A., and Esko, J.D. 2002. Heparin's antiinflammatory effects require glucosamine 6-O-sulfation and are mediated by blockade of L- and P-selectins. J. Clin. Invest. 110:127-136. doi:10.1172/JCI200214996.

60. Varki, N.M., and Varki, A. 2002. Heparin inhibition of selectin-mediated interactions during the hematogenous phase of carcinoma metastasis: Rationale for clinical studies in humans. Semin. Thromb. Hemost. 28:53-66.

61. Beeley, J.G. 1985. Glycoprotein and proteoglycan techniques. Elsevier. New York, New York, USA. 73-78.

62. Manzi, A.E., Diaz, S., and Varki, A 1990. High-pressure liquid chromatography of sialic acids on a pellicular resin anion-exchange column with pulsed amperometric detection: a comparison with six other systems. Anal. Biochem. 188:20-32.

63. Corriveau, D., and Fritsma, J. 1988. Hemostasis and thrombosis in the clinical laboratory. 1988. J.P. Lippincott Co. Philadelphia, Pennsylvania, USA. 443 pp.
64. Lehr, H.A., Mankoff, D.A., Corwin, D., Santeusanio, G., and Gown, A.M. 1997. Application of Photoshop-based image analysis to quantification of hormone receptor expression in breast cancer. J. Histochem. Cytochem. 45:1559-1565.

65. Capon, C., et al. 1997. Sulfated Lewis X determinants as a major structural motif in glycans from LS174T-HM7 human colon carcinoma mucin. J. Biol. Chem. 272:31957-31968.

66. Moesby, L., Hansen, E.W., and Christensen, J.D. 2000. Endotoxin testing of proteins for parenteral administration using the Mono Mac 6 assay. J. Clin. Pharm. Ther. 25:283-289.

67. Tian, F., Appert, H.E., Myles, J., and Howard, J.M. 1992. Prognostic value of serum CA 19-9 levels in pancreatic adenocarcinoma. Ann. Surg. 215:350-355.

68. Harmenberg, U., Wahren, B., and Wiechel, K.L. 1988. Tumor markers carbohydrate antigens CA 19-9 and CA-50 and carcinoembryonic antigen in pancreatic cancer and benign diseases of the pancreatobiliary tract. Cancer Res. 48:1985-1988.

69. Bevilacqua, M.P., Stengelin, S., Gimbrone, M.A., and Seed, B. 1989. Endothelial leukocyte adhesion molecule 1: an inducible receptor for neutrophils related to complement regulatory proteins and lectins. Science. 243:1160-1165.

70. Li, N., et al. 2000. Platelet-leukocyte cross talk in whole blood. Arterioscler. Thromb. Vasc. Biol. 20:2702-2708.

71. Laudanna, C., et al. 1994. Sulfatides trigger increase of cytosolic free calcium and enhanced expression of tumor necrosis factor-alpha and interleukin-8 mRNA in human neutrophils. Evidence for a role of L-selectin as a signaling molecule. J. Biol. Chem. 269:4021-4026.

72. Waddell, T.K., Fialkow, L., Chan, C.K., Kishimoto, T.K., and Downey, G.P. 1994. Potentiation of the oxidative burst of human neutrophils. A signaling role for L-selectin. J. Biol. Chem. 269:18485-18491.

73. Waddell, T.K., Fialkow, L., Chan, C.K., Kishimoto, T.K., and Downey, G.P. 1995. Signaling functions of L-selectin. Enhancement of tyrosine phosphorylation and activation of MAP kinase. J. Biol. Chem. 270:15403-15411.

74. Brenner, B., et al. 1996. L-selectin activates the Ras pathway via the tyrosine kinase p56lck. Proc. Natl. Acad. Sci. U. S. A. 93:15376-15381.

75. Malhotra, R., and Bird, M.I. 1997. L-selectin: a novel receptor for lipopolysaccharide and its potential role in bacterial sepsis. Bioessays. 19:919-923.

76. Crockett-Torabi, E., Sulenbarger, B., Smith, C.W., and Fantone, J.C. 1995. Activation of human neutrophils through L-selectin and Mac-1 molecules. J. Immunol. 154:2291-2302.

77. Crockett-Torabi, E., and Fantone, J.C. 1997. L-selectin stimulation of canine neutrophil initiates calcium signal secondary to tyrosine kinase activation. Am. J. Physiol. Heart. Circ. Physiol. 272:H1302-H1308.

78. Simon, S.I., et al. 1999. Signaling functions of L-selectin in neutrophils: alterations in the cytoskeleton and colocalization with CD18. J. Immunol. 163:2891-2901.

79. Wize, J., Sopata, I., Smerdel, A., and Maslinski, S. 1998. Ligation of selectin $\mathrm{L}$ and integrin CD11b/CD18 (Mac-1) induces release of gelatinase B (MMP-9) from human neutrophils. Inflamm. Res. 47:325-327.

80. Smolen, J.E., et al. 2000. L-selectin signaling of neutrophil adhesion and degranulation involves $\mathrm{p} 38$ mitogen-activated protein kinase. J. Biol. Chem. 275:15876-15884.

81. Hidari, K.I., Weyrich, A.S., Zimmerman, G.A., and McEver, R.P. 1997. Engagement of P-selectin glycoprotein ligand-1 enhances tyrosine phosphorylation and activates mitogen-activated protein kinases in human neutrophils. J. Biol. Chem. 272:28750-28756.

82. Evangelista, V., et al. 1999. Platelet/polymorphonuclear leukocyte interaction: P-selectin triggers protein-tyrosine phosphorylation-dependent CD11b/CD18 adhesion: Role of PSGL-1 as a signaling molecule. Blood. 93:876-885.

83. Marcus, A.J. 1990. Stratton lecture 1989. Thrombosis and inflammation as multicellular processes: pathophysiologic significance of transcellular metabolism. Blood. 76:1903-1907.

84. De Gaetano, G., Cerletti, C., and Evangelista, V. 1999. Recent advances in platelet-polymorphonuclear leukocyte interaction. Haemostasis. 29:41-49.

85. Celi, A., et al. 1994. P-selectin induces the expression of tissue factor on monocytes. Proc. Natl. Acad. Sci. U. S. A. 91:8767-8771.

86. Giesen, P.L., and Nemerson, Y. 2000. Tissue factor on the loose. Semin. Thromb. Hemost. 26:379-384.

87. Osterud, B. 2001. The role of platelets in decrypting monocyte tissue factor. Semin. Hematol. 38(Suppl. 12):2-5.

88. Zimmerman, G.A., McIntyre, T.M., Prescott, S.M., and Stafforini, D.M. 2002. The platelet-activating factor signaling system and its regulators in syndromes of inflammation and thrombosis. Crit. Care Med. 30(Suppl.):S294-S301.

89. Merten, M., and Thiagarajan, P. 2001. Role for sulfatides in platelet aggregation. Circulation. 104:2955-2960. 